\title{
CONCEPCIÓN INSTITUCIONAL DEL DERECHO A LA SALUD EN VENEZUELA*
}

\author{
INSTITUTIONAL CONCEPTION OF THE RIGHT \\ TO THE HEALTH IN VENEZUELA
}
CONCEPCIÓN INSTITUTIONNELLE DU DROIT
À LA SANTÉ EN VENEZUELA

Andy Delgado Blanco**

\begin{abstract}
RESUMEN: El propósito de este artículo es examinar la concepción del derecho a la salud que subyace en la Constitución de la República Bolivariana de Venezuela y su posterior desarrollo a través de algunas estructuras y mecanismos institucionales puestas en práctica desde 1999 hasta 2013. Para cumplir con ese objetivo este trabajo discurrirá a lo largo de dos cauces: las propuestas y debates suscitados en el seno de la Asamblea Nacional Constituyente de 1999 que influyeron en la cristalización de las normas constitucionales que garantizan el derecho a la salud; y los mecanismos y estructuras institucionales a través de los cuales se ha venido desarrollando el derecho. La revisión documental realizada sugiere que aun cuando las normas constitucionales postulan un derecho a la salud con un carácter marcadamente universalista; en los hechos, por una parte, las estructuras y mecanismos institucionales desarrollados son poco transparentes y repiten esquemas pasados; por la otra, el creciente deterioro de las condiciones de acceso a servicios, tratamientos y medicamentos evidencia la ausencia de garantías reales para hacer efectivo el derecho.
\end{abstract}

Palabras claves: derecho a la salud, Constitución, Venezuela.

\footnotetext{
* Recibido el 13 de junio de 2017 y aprobado para su publicación el 4 de septiembre de 2017.

** Centro de Estudios del Desarrollo. Universidad Central de Venezuela.
}

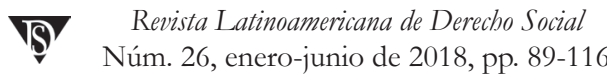


ABSTRACT: The purpose of this article is to examine the conception of the right health expressed in the Constitution of Bolivarian Republic of Venezuela and its further development through some structures and institutional mechanisms implemented from 1999 until 2013. In order to achieve that objective this work shall proceed along two tracks: the proposals and discussions provoked within of the National Constituent Assembly of 1999 that influenced the crystallization of the constitutional norms that ensuring the right to the health and the mechanisms and institutional structures across which one has come developing the right. The documentary review suggests that even if the constitutional rules postulate a right to the health with a pronounced universalist character; in the facts, on one hand, the structures and institutional mechanisms implemented are not transparent and repeat schemes of the past; and for other one, the increasing deterioration of the conditions of access to services, treatments and medicines illustrate the lack of royal guarantees to make the right effective.

Keys words: right to the health, Constitution, Venezuela

RÉSUMÉ: Le propos de cet article est d'examiner la conception du droit à la santé qui se sous-trouve dans la Constitution de la République bolivarienne du Venezuela et son développement postérieur à travers de quelques structures et mécanismes institutionnels mises en pratique de 1999 à 2013. Pour réalisé de cet objectif ce travail il réfléchira le long de deux pistes: les propositions et les débats suscités dans le cadré de l'Assemblée Nationale Constituante de 1999 qui ont influé sur la cristallisation des normes constitutionnelles qui garantissent le droit à la santé es propositions qui ont influé sur la cristallisation des normes constitutionnelles qui garantissent le droit à la santé et les mécanismes et les structures institutionnelles à travers desquels s'est développée le droit. La révision documentaire réalisée suggère que même si les normes constitutionnelles postulent un droit à la santé avec un caractère nettement universaliste; dans les faits, d'une part, les structures et les mécanismes institutionnels développés manquent de transparence et répètent des schémas passes, par l'autre, la détérioration croissante des conditions de l'accès aux services, les traitements et les médicaments met en évidence l'absence de garanties réelles pour faire le droit effectif.

Mots-clés: droit á la santé, Constitution, Venezuela. 
SUMARIO: I. Nota introductoria. II.Contexto institucional de los derechos sociales en la Venezuela del siglo XX. III. El derecho a la salud en la Constitución de 1999. Propuestas y debates. IV. Desarrollo del derecho a la salud. Estructuras y mecanismos institucionales. V. A manera de cierre. VI. Referencias.

\section{NOTA INTRODUCTORIA}

L

os referentes institucionales tienen un peso específico en la forma en que se desarrollan las normas que rigen la vida de una sociedad determinada. En el caso del derecho a la salud las reglas, mecanismos, instancias, contextos, recursos y actores son claves para incidir tanto en su definición como en su desarrollo; este derecho tiene una relación de sincronía con aspectos ligados a la integración social, la solidaridad, el consumo e incluso con la inserción laboral y la organización del trabajo: en la medida en que la población goza de mejores condiciones de salud aumenta su calidad de vida lo que, entre otras razones, se traduce en ciudadanos con mayor disposición al trabajo y una disminución de la cantidad de ausencias laborales por enfermedad.

En Venezuela la Constitución de 1999 consagró de manera expresa la salud como uno de los derechos sociales, parte integrante del derecho a la vida; le adjudicó al Estado el rol de garante y promotor de políticas orientadas a elevar la calidad de vida, el bienestar colectivo y el acceso a los servicios; también estableció como deber de los ciudadanos su activa participación en la promoción y defensa del derecho.

Descubrir el tono e intencionalidades de las proposiciones que se hicieron ante la comisión responsable de redactar los artículos que, en materia de salud, se llevarían a la plenaria de la Asamblea Nacional Constituyente de 1999, examinar las discusiones que los diferentes actores políticos sostuvieron, los mecanismos y políticas instrumentadas para activar el derecho, forman parte del próposito de este artículo, tal como se expondrá en las páginas que siguen.

\section{CONTEXTO INSTITUCIONAL DE LOS DERECHOS SOCIALES EN LA VENEZUELA DEL SIGLO XX}

Los procesos sociopolíticos no son aislados y se ven influenciados por múltiples factores, de allí lo significativo que resulta revisar sumariamente la 
evolución y ampliación de los derechos sociales en Venezuela, y muy especialmente la del derecho a la salud, hasta llegar a su situación actual.

Los beneficios y derechos de contenido social tienen un tímido surgimiento en Venezuela a partir de 1936, con la muerte del dictador Juan Vicente Gómez, y se van expandiendo lenta y progresivamente durante toda la década de los cuarenta. Es en estos años cuando se crea un cuerpo constitucional y legal sobre la protección laboral y la seguridad social, una red institucional a través del Instituto Nacional de Higiene, el Ministerio de Sanidad y Asistencia Social, y el Instituto Venezolano de los Seguros Sociales, entre otros. La explotación petrolera sirvió de marco para mejorar las condiciones de vida de la población y variar el mapa sanitario del país: fue erradicado el paludismo que diezmaba la población, el sistema médico asistencial y de carreteras comenzó a desarrollarse; se construyeron vías de penetración para hacer accesible el camino a los pozos petroleros y comunicar los centros urbanos entre sí; se formaron los primeros sindicatos petroleros y los partidos modernos.

En materia constitucional destaca la carta magna que, imbuida por las Constituciones de Querétaro de 1917, de Weimar de 1919 y la española de 1931, vio la luz en 1947. Este instrumento vino a representar un notable avance para la época, con su enfoque centrado en un modelo societario igualitario y en la justicia social; institucionalizó como obligación del Estado, en el plano individual y colectivo, los derechos de los trabajadores a la salud y a la seguridad social. Pese a su corta vigencia debido a un gobierno de facto que subvirtió el orden constitucional apenas meses después de su promulgación, la Constitución de 1947 tendría una notable influencia una vez restaurado el hilo constitucional, hacia finales de los años cincuenta.

Producto de la voluntad política de los actores más influyentes de la época, expresada en el Pacto de Punto Fijo' ${ }^{1}$ en 1961 se promulgó una nueva Cons-

\footnotetext{
1 Se conoce como Pacto de Punto Fijo al acuerdo firmado el 31 de octubre de 1958, recién salido el país de la larga dictadura de Marcos Pérez Jiménez, por los dirigentes de tres de los partidos que habían estado presentes en el proceso constitucional en 1947, es decir, $\mathrm{AD}$, Copei y URD, quienes acordaron el rol institucional de los actores en el nuevo modelo democrático: el Estado tendría un papel central en casi todos los ámbitos de la nación; el sector privado, uno secundario en la activación de la economía y, los partidos vendrían a ser los articuladores o intermediarios entre el Estado y los ciudadanos. El Partido Comunista fue excluido de la firma — no del sistema democrático — por cuanto se consideró que sus propuestas programáticas eran incompatibles con el modelo de hegemonía que subyacía en el acuerdo.
} 
titución que dio continuidad al ideario constitucional de 1947: un modelo igualitario de sociedad expresado en un Estado social de derecho que estuvo vigente hasta finales de 1999. La Constitución de 1961 obligó a desarrollar un sistema de seguridad social universal y contributivo en paralelo con uno asistencial no contributivo; se avanzó en la protección social de la población al tiempo que se limitó, formalmente, la práctica asistencialista únicamente a los que careciesen de recursos económicos. El progreso y modernización del país se manifestaba en infraestructura y satisfacción de demandas mediante la prestación de servicios públicos, comenzó a perfilarse una nueva sociedad: las grandes masas se movilizaron aún más hacia los centros urbanizados y se aceleró el proceso de industrialización.

Más adelante, ya en los setenta, la crisis que azotó la región golpeó al país apareciendo los primeros desajustes socioeconómicos y fiscales que se irían agudizando hasta hacer eclosión hacia finales de los ochenta y parte de los noventa, con lo cual, el deformado aparato estatal no pudo seguir dando respuestas a las demandas sociales de la población. En materia de salud, el modelo institucional del sector lucía desconectado, disperso, agotado e incapaz de impactar en las condiciones de vida de la mayoría de la población al limitarse a las actividades curativas e individuales, con un escaso radio de acción hacia lo preventivo-colectivo. ${ }^{2}$

Los llamados y alertas para satisfacer los requerimientos sociales, económicos, fiscales, modificar la estructura estatal y reformar la Constitución no fueron atendidos debidamente y el país siguió transitando por cauces peligrosos: mientras las clases dirigentes perdían el favor de las populares, se asistió a un estallido social, dos fallidos golpes de Estado, y la renuncia de un presidente de la República acusado por actos de corrupción, todo ello en medio de promesas de cambios a la institucionalidad y un intento de reforma constitucional que no prosperó. Fue ya en el año noventa y nueve, previa convocatoria de la última Asamblea Nacional Constituyente del siglo pasado, cuando se discutió y aprobó un nuevo texto constitucional.

Voces y miradas diversas y plurales de ciudadanos organizados y no organizados, grupos de trabajo, comunidades, academias, universidades, entes oficiales, sindicatos, partidos políticos expusieron sus ideas sobre los temas a discutirse en esa Asamblea. En el caso de la salud el abanico de propuestas abarcó

2 Comisión Presidencial para la Reforma del Estado, Una politica social para la reafirmación de la democracia, Caracas, Editorial Arte, 1989, vol. 8. 
desde su concepción y el derecho que la garantiza, hasta la forma en que debían prestarse los servicios, las características del sistema y la responsabilidad de la sociedad, incluido el Estado. Como resultado de este proceso se consagró constitucionalmente una visión amplia de la salud como parte del derecho a la vida, y por ende, como un derecho humano que debía realizarse y hacerse efectivo con el Estado jugando un rol central y los ciudadanos como contralores.

\section{EL DERECHO A LA SALUD EN LA CONSTITUCIÓN DE 1999 PROPUESTAS Y DEBATES}

En diciembre de 1998 llegó al poder en Venezuela, por vía electoral, la cabeza visible del primer intento de derrocamiento del entonces presidente constitucional de la República, Sr. Carlos Andrés Pérez, ocurrido seis años antes. La promesa central que marcó la campaña del candidato, a la postre ganador de la presidencia, fue la convocatoria de una Asamblea Constituyente que sentara las bases de una nueva República, lo que efectivamente hizo el mismo día de su juramentación como presidente al firmar un decreto presidencial para “...la realización de un referendo para que el pueblo se pronunciara sobre la convocatoria de una Asamblea Nacional Constituyente". ${ }^{3}$

Producto de la Asamblea Nacional Constituyente se discutió, redactó y promulgó la Constitución de la República Bolivariana de Venezuela que estableció cambios institucionales y normativos de notable trascendencia, entre los que se cuentan: la ampliación de los derechos sociales, la cristalización de la salud como un derecho humano fundamental y el establecimiento de la corresponsabilidad ciudadana. Ese proceso de reivindicación de la concepción redistributiva de los derechos sociales obedeció, en gran medida, a los esfuerzos de la sociedad venezolana quien se volcó a los espacios públicos, a través de individualidades y ciudadanos organizados, para tratar de incidir en el debate constituyente, como se verá en el acápite siguiente.

\section{Propuestas}

Hecha la convocatoria para elegir a los representantes a la Asamblea Nacional Constituyente, en paralelo al debate electoral, se despertó en el país un

\footnotetext{
3 Decreto de la Presidencia de la República, núm. 3, 1999.
} 
amplio y espontáneo proceso participativo. Ciudadanos, grupos de diversa índole y organizaciones pertenecientes a la sociedad civil, se dieron a la tarea de exponer sus ideas sobre los temas a discutirse. La Asamblea Nacional Constituyente, instalada el 3 de agosto de 1999, se estructuró en veinte comisiones de trabajo, las cuales elaboraron por separado las propuestas de artículos de las materias a regular y las enviaron a la Comisión Constitucional quien se encargó de integrar lo recibido en un proyecto que luego sería discutido en las plenarias llevadas a cabo en el seno de la Asamblea.

Una de esas comisiones fue la de los derechos sociales y de la familia, salud, mujer, infancia, ancianos y discapacitados que tenía como objetivo: “....redactar los artículos que expresaran una visión de salud consonante con la nueva Venezuela, con los conceptos fundamentales y centrales de la nueva Constitución y con la redefinición del papel del Estado y los ciudadanos en la sociedad". ${ }^{4}$ Esta Comisión se estructuró en tres subcomisiones: a) Familia y Mujer, b) Derechos Laborales y Seguridad Social, y c) Salud. El trabajo de esta última subcomisión se realizó en tres momentos; en el primero se hizo un diagnóstico de la situación del sector salud en el país; en el segundo se realizó una investigación documental sobre la salud en las constituciones de todos los países de América Latina y algunos de Europa y Norte América; el tercero estuvo constituido por la participación de diversos sectores de la sociedad venezolana, los cuales expusieron en el seno de la Subcomisión sus iniciativas en la materia. ${ }^{5}$

La Subcomisión de salud tuvo 16 sesiones abiertas a todo el público, acordó 100 derechos de palabra y recibió por escrito 80 propuestas ${ }^{6}$ provenientes de organizaciones no gubernamentales, cooperativas, academias y universidades, sindicatos, cámaras privadas ligadas al sector, ciudadanos particulares, partidos políticos, grupos de trabajo y comunidades que espontáneamente se reunieron para discutir su visión de la salud y de entes oficiales, bien a través de sus representantes y técnicos o indirectamente, como de consultas ciudadanas promovidas en las diversas entidades federales del país.

4 Feo, Oscar, "La salud en la nueva Constitución", en Maingon, Thais, (coord.), La cuestión social en la constitución bolivariana de Venezuela, Caracas, Cendes-UCV, 2000, serie Temas de Docencia, pp. 29-46.

5 Feo, Oscar y Pascualina Curcio, "La salud en el proceso constituyente venezolano", Revista Cubana Salud Pública, La Habana, vol. 30, núm. 2, junio de 2004, disponible en: wnm.scielo. sld.cu, consultado el 29 mayo de 2012.

6 Feo, Oscar, "La salud en la nueva Constitución", op. cit. 
Las visiones presentadas fueron tan diversas como las personas y grupos participantes, no todas tenían un propósito claro y definido sobre el derecho a la salud; los pronunciamientos hechos abarcaron un amplio espectro y trataban sobre el sistema de salud que debía crearse, la salud pública y otros temas como la salud reproductiva, salud holística, medios naturales para la prevención y recuperación de la salud; sin embargo, para los efectos de esta investigación, aun cuando se revisararon todos y cada uno de los documentos que reposan en los archivos, sólo se tomaron en cuenta 53 propuestas. Todas referidas, de una u otra manera, a la concepción del derecho a la salud y los medios para hacerlo efectivo: desde las expectativas y aspiraciones ciudadanas sobre las formas y mecanismos de funcionamiento de un sistema de salud, hasta las que hicieron hincapié en la concepción del derecho a la salud. Las más influyentes, en cuanto a fondo y forma, vinieron de las comunidades, academias, organizaciones de derechos humanos y entes oficiales, en las que se advierte el influjo de los organismos internacionales y corrientes doctrinarias en la materia. Éstas coincidían en una visión de la salud como derecho basado en principios y garantías que lo hicieran efectivo así como, en la participación de los ciudadanos en el control y vigilancia de las políticas en la materia. Como resultado de esa conjución la Subcomisión de Salud presentó a la Comisión Constitucional una propuesta de ocho artículos que finalmente fue reducida a tres, los cuales pasaron a formar parte del proyecto de constitución a discutirse en la plenaria y que estaban referidos a la salud como derecho, el sistema de salud a regir, y su forma de financiamiento.

\section{Debate constitucional}

Al contrario de lo que sucedía en las calles del país e incluso a lo interno de las veinte comisiones y sus respectivas subcomisiones, las plenarias de la Asamblea se caracterizaron por una exigua discusión, lo que pudiera explicarse en los llamados públicos del presidente de la República para culminar su cometido antes de los seis meses previstos. ${ }^{7}$ Los constituyentes discutieron el

7 De acuerdo con las bases comiciales establecidas por el Consejo Nacional Electoral, la Asamblea debía funcionar durante 180 días contados a partir de su instalación. Sin embargo, el nuevo texto constitucional fue sancionado cuatro meses después; luego, vendría su 
proyecto de Constitución en medio de gran apremio entre el 20 de octubre y el 25 de noviembre de 1999: la primera discusión se hizo en forma "inusitadamente rápida y con celeridad casi irracional"8 en 19 plenarias; mientras que la segunda, en sólo tres, entre el 12 y el 14 de noviembre. La única discusión de los derechos sociales, incluído el derecho a la salud, se produjo el sábado 30 de octubre de 1999.

El proyecto recogía una concepción centrada en la salud como un derecho social parte del derecho a la vida, responsabilidad del Estado, quien debía garantizarla, en condiciones de igualdad, a todos los ciudadanos. Se asentaron, igualmente, tres aspectos que buscaban dar efectividad de esa garantía: la centralidad del Estado como órgano rector de las políticas de salud; la caracterización del sistema como gratuito, único e intersectorial; así como la función contralora y participativa del ciudadano en favor de la eficacia de las acciones estatales. ${ }^{9}$

El debate sobre el derecho a la salud se inició con la lectura del que habría de ser el artículo 87 de la Constitución. ${ }^{10}$ Se recogía el espíritu de las sugerencias hechas por los ciudadanos organizados: la salud es un derecho social y el objeto a tutelar la salud como tal. Este último asunto suscitó algunas discrepancias, entre las que destacan las del constituyente Allan Brewer-Carías, influyente académico proveniente del mundo del derecho, quien cuestionó dos aspectos; por una parte, consideró que la salud como tal es imposible de garantizar y por la otra, se mostró en desacuerdo con dejar al Estado la exclusividad en la responsabilidad por la tutela de la salud. Su primer cuestionamiento se sustentó en que esa responsabilidad ha de dirigirse hacia la protección de la salud a través de las políticas y medios necesarios; ${ }^{11}$ ya que es

ratificación mediante referéndum popular del 15 de diciembre y su promulgación el 30 de diciembre de ese mismo año.

8 Brewer-Carías, Allan, La Constitución de 1999, Caracas, Editorial Arte, 2000, p. 35.

9 Feo, Oscar, op. cit.

10 La norma propuesta a la plenaria era del tenor siguiente: Artículo 87. "La salud es un derecho social fundamental, responsabilidad instransferible del Estado, quien lo garantiza como parte del derecho a la vida. Ese Estado promoverá y desarrollará políticas orientadas a elevar la calidad de vida, el bienestar colectivo y el acceso a los servicios. Todas las personas tienen derecho a la protección de la salud, el deber de participar activamente en promoverla y defenderla, y cumplir con las medidas sanitarias y de saneamiento que establezca la ley de conformidad a los tratados y convenios internacionales". Asamblea Nacional Constituyente, Debates de la Constitución de 1999 (versión en corrección), Caracas, Servicio Autónomo de Información Legislativa, 1999, p. 24.

11 Idem. 
imposible evitar que alguien se enferme y menos aun garantizar sempiternamente la salud de los ciudadanos; argumentó igualmente que la tutela de la salud no debía ser exclusiva del Estado sino que debía ser una tarea compartida.

La responsabilidad de la garantía del derecho se mantuvo presente a lo largo del debate. Para el constituyente Leopoldo Puchi ${ }^{12}$ en la propuesta se desdibujaban los límites entre responsabilidad y garantía; la primera debía ser compartida y la segunda, exclusiva del Estado. Ambos actores, sociedad y Estado, debían trabajar juntos, en materia de salud, sin que ello significara que el último de los mencionados dejara de ser el responsable de garantizar el derecho. Al término de esta intervención el presidente de la Asamblea pidió a una destacada miembro del Polo Patriótico un pronunciamiento sobre las intervenciones hechas hasta ese momento, quien argumentó que el artículo en cuestión ya había sido suficientemente debatido y debía dejarse “...para una segunda discusión... volver si es posible sobre el tema...” (cursivas de quien escribe). ${ }^{13}$ Luego de esta intervención el asunto fue sometido a votación y aprobado. En la segunda discusión no hubo análisis ni controversia ya que los constituyentes se limitaron a aprobar los artículos luego de su lectura a la Asamblea.

Como puede colegirse de las transcripciones comentadas la garantía del derecho, la responsabilidad del Estado y la participación ciudadana estuvieron muy presentes en los debates, al igual que, el rol estatal en la gestión del sistema de salud. Algunos de los constituyentes se opusieron a darle un carácter de exclusividad al Estado e impedir la participación de la sociedad; otros se mostraron en contra de la privatización de los servicios de salud y a favor de un rol principalísimo del Estado en su gestión y garantía. En las disposiciones aprobadas se eliminó la idea inicial del Estado como único responsable de la gestión de los programas de salud y se cerró la puerta a la posibilidad de privatizar los servicios públicos de salud.

El contenido de las discusiones sobre la concepción del derecho a la salud y las garantías para hacerlo efectivo está recogido, en esencia, en las líneas anteriores. En ningún caso se permitió agregado o añadido alguno sobre los textos que habían sido traídos al debate, aun cuando a posteriori varió la re-

12 Representante de la coalición gubernamental y ex ministro del gabinete del presidente Chávez.

13 Asamblea Nacional Constituyente, op. cit. 
dacción de los artículos. Como resultado de este proceso, en el capítulo V, "De los derechos sociales y de las familias" de la Constitución aprobada, se delineó una norma que garantiza la salud como un derecho social, parte integrante del derecho a la vida:

Artículo 83. La salud es un derecho social fundamental, obligación del Estado, que lo garantizará como parte del derecho a la vida. El Estado promoverá y desarrollará políticas orientadas a elevar la calidad de vida, el bienestar colectivo y el acceso a los servicios. Todas las personas tienen derecho a la protección de la salud, así como el deber de participar activamente en su promoción y defensa, y el de cumplir con las medidas sanitarias y de saneamiento que establezca la ley, de conformidad con los tratados y convenios internacionales suscritos y ratificados por la República. ${ }^{14}$

El sujeto protegido por el derecho está constituido por todas las personas, venezolanas o no, ciudadanas o no del Estado venezolano, a quienes se les atribuye, el rol expreso de garantizar el derecho. En paralelo, se prescribe la activa participación de los ciudadanos en su promoción y defensa, así como la obligación que tienen de someterse a las medidas sanitarias y de saneamiento que establezca la ley. El objeto tutelado es la salud, la promoción y el desarrollo de políticas orientadas a elevar la calidad de vida, el bienestar colectivo y el acceso a los servicios, para lo cual el Estado deberá utilizar como medios las políticas sociales, las medidas sanitarias y de saneamiento a que hubiere lugar.

Además del precitado artículo 83 en el que se consagra un derecho ilimitado, dirigido a todos, independientemente de los medios de fortuna que pudieran poseerse; la Constitución recoge otras dos disposiciones ${ }^{15}$ en las que se deja claro que la satisfacción de ese objetivo y los medios para lograrlo son responsabilidad del Estado, quien debe cumplir con su compromiso a través de un sistema público nacional de salud, integrado al sistema de seguridad social y caracterizado por ser intersectorial, descentralizado, participativo, gratuito, universal, integral, equitativo, integrado y solidario, cuyo financiamiento, también, es su obligación.

Más allá de la norma, la realización de un derecho suele depender de la naturaleza, calidad, intensidad, oportunidad y pertinencia de las reglas, valores,

14 Constitución de la República Bolivariana de Venezuela.

15 Artículos 84 y 85. 
principios, estructuras y mecanismos que se desarrollen para hacerlo efectivo; en este sentido, en la próxima sección se discutirá lo relativo a la realización efectiva del derecho a la salud en Venezuela, con especial enfasis en lo sucedido luego del proceso constituyente ya antes aludido.

\section{Desarrollo DEL DEREChO A LA SALUd.}

\section{ESTRUCTURAS Y MECANISMOS INSTITUCIONALES}

Las políticas sociales instrumentadas en Venezuela, a partir de los años sesenta del siglo pasado, tuvieron un corte tradicional y pudieran caracterizarse como universales, dirigidas a vincular la intervención social del Estado con las bases de apoyo al sistema político, centradas en un sistema de seguros sociales para proteger a los trabajadores y con énfasis en la gratuidad de la educación y en los servicios de salud. En ese marco hubo subsidios a la vivienda, al transporte y a los productos de consumo masivo; se buscaba intervenir tanto el mercado laboral como los precios de los artículos básicos de consumo. ${ }^{16}$ En materia de salud, el rasgo que va a marcar la política pública desde el primer tercio del siglo pasado hasta su casi finalización es su carácter eminentemente preventivo, sustentado en la atención hospitalaria; será ya en los ochenta cuando esto se revertirá y el énfasis estará tanto en las actividades de carácter curativo, como en los recortes a las inversiones en el sector y el fortalecimiento al sector privado; en los noventa, el foco estuvo en la atención en los grupos más desasistidos y se empezó a perder, en los hechos, su carácter universal. ${ }^{17}$

16 González, Lissette, La politica social en Venezuela, Caracas, Fundación Centro Gumilla, 1996, Curso de Formación sociopolítica, núm. 35.

17 Para seguir esta discusión véase, entre otros, a Castellanos, Pedro L., "Notas sobre el Estado y la salud en Venezuela", Cuadernos de la sociedad venezolana de planificación, Caracas, núm. 156-158, 1982, pp. 69-152; Kornblith y Maingon, Estado y gasto público en Venęuela, 1936-1980, Caracas, Ediciones de la Biblioteca de la Universidad Central de Venezuela, 1985; Márquez, El Estado social en Venezuela, Caracas, Ediciones del Congreso de la República, 1992; Méndez, Estado, politica socialy trabajo social en la Venezuela actual, Caracas, Universidad Central de Venezuela, Facultad de Ciencias Económicas y Sociales, Unidad de Publicaciones, 1992; Convite y Observatorio Comunitario por el Derecho a la Salud, Informe sobre el derecho a la salud en Venezuela 2007. Situación del derecho a la atención sanitaria, 2007; Uharte, Luis, Política social en Venezuela: ¿un nuevo paradigma?, tesis doctoral, Universidad Complutense de Madrid, 2008; Maingon, Thaís, "Política social en Venezuela: 1999-2003”, Cuadernos del Cendes, Caracas, núm. 55, 2004, pp. 47-73. 
Con el agotamiento de ese modelo de desarrollo ${ }^{18}$ estas políticas fueron perdiendo su carácter universal y adquirieron una función subsidiaria de las económicas: se les utilizaba para compensar los efectos dejados por la instrumentación de medidas fiscales para superar la crisis, sin que las expectativas y demandas de numerosos sectores de la población fuesen satisfechas.

Al iniciar su mandato en 1999, el presidente Chávez propuso una política social de carácter integral para ampliar las capacidades productivas de la sociedad venezolana y superar la pobreza. Meses más tarde, luego de la entrada en vigencia de la Constitución se diseñaron los primeros lineamientos políticos, jurídicos, sociales y organizacionales para estructurar y construir un nuevo proyecto nacional que, a juicio de D’Elía se basó en tres ejes centrales: la construcción de una ciudadanía social cimentada en la universalidad de los derechos sociales, la búsqueda de la equidad como objetivo supremo del ordenamiento económico y social, y por último, el rescate de lo público como espacio para el ejercicio de una verdadera democracia, lo que debía traducirse en la participación de todas las personas en los asuntos de interés público. ${ }^{19}$

La política social de estos primeros años se estructuró a partir elementos formales, discursivos y reales, no siempre coherentes entre sí. En el papel y las formas, hubo una orientación explícita hacia la persona humana y el ciudadano, la revalorización de lo social, la incorporación de la noción de capital humano y social; se enfatizó en la integración, participación social y corresponsabilidad, todo en el marco formal de la democracia participativa y protagónica delineada en la Constitución, lo que sirvió de pábulo para la formación de organizaciones que favorecieran la articulación de los diferentes actores, públicos, privados, individuales y colectivos. ${ }^{20}$

18 Modelo ISI: Inversión por sustitución de importaciones.

19 D’Elía, Yolanda, “Cambiando la orientación de las políticas públicas hacia el impacto en la calidad de vida desde una perspectiva de derechos y equidad", Informe Social, Caracas, IldisFundación Friedrich Ebert, núm. 7, 2002, pp. 10-14.

20 Véase entre otros, Alvarado, Neritza, "Populismo, democracia y política social en Venezuela", Fermentum, Mérida, año 15, núm. 44, septiembre-diciembre de 2005, pp. 305-331; Alvarado, Neritza, "Pobreza y exclusión en Venezuela a la luz de las Misiones Sociales (20032004)", Fermentum, Mérida, año 14, núm. 39 , enero-abril de 2004, pp. 181-232; Alvarado, Neritza, "La atención a la pobreza en Venezuela del 'gran viraje' a la 'V República', 1989-2002", Revista Venezolana de Análisis de Coyuntura, vol. IX, núm. 2, julio-diciembre de 2003, pp. 111-150; Alvarado, Neritza. "Pobreza y asistencialismo en Venezuela", Revista de Ciencias Sociales, vol. IX, diciembre de 2003, pp. 431-458. 
En paralelo a esas líneas principistas se continuó alimentando un discurso de condena a las políticas ejecutadas durante el gobierno anterior y que ya se había hecho presente en la campaña electoral, cuando el entonces candidato Chávez "prometió que su gestión se caracterizaría por la fórmula F=2SE: dos moléculas de política social y 1 de política económica"; 21 no obstante, en los hechos, al menos hasta 2001, se mantuvieron nueve de los catorce programas de la Agenda Venezuela y se conservaron "las estrategias asistencialescompensatorias-focalizadas anteriores... pese a su insuficiencia para mitigar la pobreza y los déficits sociales". 22

Las primeras acciones gubernamentales se asentaron básicamente en el Plan de Desarrollo Económico y Social de la Nación 2001-2007.23 Uno de sus polos, el denominado "equilibrio social”, establecía que el norte de la política social era la justicia social, objetivo macro que se dividía en tres subobjetivos: 1) garantizar el disfrute de los derechos sociales de forma universal y equitativa, 2) mejorar la distribución del ingreso y la riqueza, 3) fortalecer la participación social y generar poder ciudadano en espacios públicos de decisión, bajo el principio de corresponsabilidad.

Progresivamente, la situación política y económica se fue complejizando: bajaron los precios del barril de petróleo en medio de un clima de polarización y crispación política, que incluyó paros, ciclos insurreccionales y un fallido golpe de Estado Venezuela volvió a asistir a rupturas institucionales, políticas y sociales; en diciembre de 2002 sectores opuestos al Ejecutivo Nacional realizaron un paro que se mantuvo hasta bien entrado el año siguiente, con consecuencias adversas para la industria petrolera y el aparato productivo del país y que, en el corto y mediano plazo, dejaría un significativo déficit fiscal y social. Pese a ello, las orientaciones de la política social se mantuvieron iguales en medio de notables incrementos del gasto social destinado a los principales programas sociales. ${ }^{24}$

21 Alvarado, Neritza, "La atención a la pobreza en Venezuela...", cit.

22 Alvarado, Neritza, "Pobreza y Exclusión en Venezuela...", cit.

23 República Bolivariana de Venezuela, Ministerio del Poder Popular para la Planificación y el Desarrollo, 2001.

24 Sobre la acción expansiva del gasto véase, entre otros, Norbis, "Estado y políticas sociales en Venezuela, ¿la Quinta República o el regreso al pasado?", Revista Venezolana de Gerencia, año 7, núm. 18, 2002, pp. 237-266; Mujica, Norbis y Neritza, Alvarado, "Pobreza y 
Para 2003 se instrumentaron las "Misiones sociales", dispositivos nacidos como una clara estrategia electoral ${ }^{25}$ para “.... reducir la exclusión social, atender las áreas de la salud, alimentación, vivienda, producción y educación” y sobre las cuales, en lo sucesivo, habría de sustentarse la política social del gobierno del presidente Chávez. En el transcurso de ese año se crearon unos 13 programas (denominados misiones) dirigidos a la inclusión social en aspectos sensibles como lo alimentario, la atención primaria en salud, lo productivolaboral, atención al campesinado, alfabetización, por sólo mencionar algunas de ellas. Así surgen las misiones: Mercal, Barrio Adentro, Robinson I y II, Ribas, Sucre, Vuelvan Caras, Milagros y Marte, entre otras, ejecutadas a través de estructuras paralelas a los esquemas institucionales conocidos hasta el momento, con fundaciones que tenían la responsabilidad de administrar los recursos suministrados por PDVSA. ${ }^{26}$

Las políticas sociales instrumentadas en estos últimos años -y dentro de ellas las de salud - se han caracterizado por incorporar nuevos actores y mecanismos de mediación entre el Estado y la sociedad; entre los primeros, se contó —al menos en una primera fase- el sector militar, la compañía estatal de petróleos venezolana y, más tarde, la Misión médica cubana, las organi-

política social en Venezuela hoy: reflexiones sobre su concepción y praxis", Revista BCV, Foros 1, Caracas, abril de 2004, pp. 145-163.

25 De acuerdo con Convite y el Observatorio Comunitario por el Derecho a la salud, esta intención fue expresada por el presidente de la República en una de sus alocuciones al país: "Ustedes deben recordar que, producto del golpe y todo el desgaste aquel, la ingobernabilidad que llegó a un grado alto, la crisis económica, nuestros propios errores, hubo un momento en el cual nosotros estuvimos parejitos, o cuidado si por debajo. Hay una encuestadora internacional recomendada por un amigo que vino a mitad del 2003, pasó como 2 meses aquí y fueron a Palacio y me dieron la noticia bomba: "Presidente, si el referéndum fuera ahorita usted lo perdería... Entonces fue cuando empezamos a trabajar con las misiones, diseñamos aquí la primera y empecé a pedirle apoyo a Fidel. Le dije: "Mira, tengo esta idea, atacar por debajo con toda la fuerza”, y me dijo: 'Si algo sé yo es de eso, cuenta con todo mi apoyo'. Y empezaron a llegar los médicos por centenares, un puente aéreo, aviones van, aviones vienen y a buscar recursos, aquí la economía mejoró, organizar los barrios, las comunidades. Aquí en Caracas empezaron con Freddy, con José Vicente allá en Sucre, en Miranda con las guarniciones militares, en aquellos estados críticos. Y empezamos a inventar las misiones" (Convite y el Observatorio Comunitario por el Derecho a la salud, Informe sobre el Derecho a la salud en Venezuela. Situación del Derecho a la Atención Sanitaria, 2007, p. 81).

26 D’Elía, Yolanda y Cabezas, Luis, Las Misiones sociales en Venezuela, Caracas, Instituto Latinoamericano de Investigaciones Sociales (Ildis), Convite A.C. 2008. 
zaciones comunitarias y políticas vinculadas al gobierno central. Las fuentes de financiamiento variaron desde la creación del llamado Fonden ${ }^{27}$ hasta la utilización de los recursos asignados directamente por la estatal petrolera. ${ }^{28}$

Los esfuerzos para diseñar e instrumentar las políticas en salud comenzaron inmediatamente después de la entrada en vigencia de la Constitución con la puesta en funcionamiento de un modelo de Atención Integral de Salud para fortalecer la red ambulatoria encargada de la atención primaria y que tenía como objetivo “... desarrollar actividades de promoción y prevención orientadas a la equidad y mejora de la calidad de vida... acercar los usuarios a las instituciones responsables de prestar el servicio, facilitando así el incremento en los niveles de accesibilidad al sistema de salud". ${ }^{29}$ Esta iniciativa fue acompañada por otras acciones gubernamentales cuyos componentes se vinculaban con el área de la salud, tales como el Plan Bolívar 2000, el Programa de Salud (Prosa) y el Plan Estratégico de Seguridad Alimentaría (PESA), por sólo mencionar algunos. ${ }^{30}$

En la medida en que transcurrían los primeros años de gobierno, el Ministerio de Salud y Desarrollo Social continuó manejando la estrategia integral de promoción de la salud que preveía la incorporación de la comunidad a través de tres figuras: los Núcleos de Atención Primaria en Salud, los Centros de Atención Primaria Integral y los Centros de Especialidades Ambulatorias. ${ }^{31}$

27 Fondo de Desarrollo Nacional S.A. Empresa creada por el gobierno nacional para optimizar la inversión productiva y social, se inserta dentro de la nueva estrategia económica y financiera del Ejecutivo nacional y se fundamenta en dos líneas básicas: 1) apalancar la recuperación económica (real y productiva) y, 2) incrementar la inversión social, véase: http:/ / wmw. fonden.gob.ve/.

28 Gómez, Irey y Alarcón, Luis, "Política social y construcción de ciudadanía en Venezuela”, Multiciencias, vol. 9, núm. 2, 2009, pp.167-175.

29 Colmenares, Isabel et al., "La política de salud en Venezuela en el gobierno de Hugo Chávez”, Saber, Venezuela, vol. 17, núm. 2, 2005, pp. 202-214.

30 El Plan Bolívar 2000 buscaba atender las emergencias sociales mediante la participación cívico-militar. El Plan pro salud, llevado a cabo por la Fuerza Armada Nacional, el Ministerio de Salud y Desarrollo Social y el Plan Bolívar 2000, con un objetivo multifuncional, perseguía diagnosticar, dar tratamiento y atención en enfermedades crónicas, y practicar intervenciones quirúrgicas en distintas áreas; así como, hacer mantenimiento y dotar las estructuras hospitalarias. El Plan Especial de Seguridad Alimentaria se creó para realizar operaciones de compra y venta de diversos rubros alimentarios.

31 González, Lissette y Lacruz, Tito, La Política social en Venezuela, Caracas, Centro Gumilla, 2008. 
Ahora bien, desde su instrumentación en 2003 el programa bandera del gobierno, en materia de salud, ha sido la Misión Barrio Adentro. ${ }^{32}$

Los orígenes de la Misión Barrio Adentro se remontan a febrero de 2003, cuando la Alcaldía de Libertador solicitó a la Embajada de Cuba en Venezuela, la colaboración de la Misión Médica Cubana, producto de lo cual se firmó el Acuerdo de Cooperación Técnica con Cuba. Esta Misión había trabajado en el país a partir de la atención humanitaria prestada como consecuencia de la vaguada ocurrida en el estado de Vargas, en diciembre de 1999 que, entre otros efectos, dejó inoperativa gran parte de la infraestructura sanitaria del estado. Desde entonces, un grupo de profesionales de la salud venidos de Cuba se había establecido en varias zonas de Venezuela para desarrollar el Programa Integral de Salud en las zonas de mayor necesidad. Como resultado del Convenio firmado, inicialmente llegó proveniente de Cuba, un equipo conformado por tres profesionales de la salud; luego les seguirían 50 médicos para instalarse en diez parroquias del municipio Libertador.

El objetivo central de la Misión Barrio Adentro es “...la implementación y coordinación institucional del Programa Integral de prestación de Atención Primaria de Salud...". ${ }^{33}$ La Misión se ha ido desarrollando en cuatro fases, la primera de ellas, Barrio Adentro I, consistió en la creación de consultorios y clínicas populares dentro de las comunidades "con poco acceso al sistema de salud convencional". ${ }^{34}$ Tuvo una etapa piloto, en el municipio Libertador de la ciudad de Caracas, entre abril y junio de 2003, para luego, extenderse masiva-

32 Farías-Suárez, Adriana y Farías de Estany, Jenny, "Participación comunitaria: otra mirada al sistema de salud venezolano", Población y salud en Mesoamérica, San José, vol. 7, núm. 1, julio-diciembre de 2009, pp.1-22; Villasana, Pedro, "De Alma Ata a Barrio Adentro. Una aproximación al sentido histórico de las metamorfosis del discurso de la participación en salud en Venezuela", en Lévy, Johanna y Malo, Miguel (eds.), De la participación en salud a la construcción del poder popular: experiencias para el debate, Maracay, IAES “Dr. Arnoldo Gabaldón, 2010, pp. 3170; Díaz, Jorge, Salud y hegemonía en Venezuela: Barrio Adentro, continente afuera, Caracas, CendesUCV, 2008; Organización Panamericana de la Salud, Barrio Adentro: derecho a la salud e inclusión social en Venezuela, Caracas, 2006; Contreras, Juana, El Plan Barrio Adentro. Ponencia presentada en el seminario nacional: política social, ¿un nuevo paradigma?, Caracas, FEGS, 2004; González, José, Misión Barrio Adentro: Programa de Atención, Inclusión y Equidad en Salud, Maracay, IAESP "Dr. Arnoldo Gabaldón” (Mimeo), 2005; Alayón, Rubén, "Barrio Adentro: combatir la exclusión profundizando la democracia”, Revista Venezolana de Economía y Ciencias Sociales, vol. 11, núm. 3, septiembre-diciembre de 2005, pp.219-244, entre otros.

33 Decreto de la Presidencia de la República, núm. 2745.

34 Comisión de Enlace para la Internacionalización de las Misiones Sociales, Misión Barrio Adentro I, II, III, IV, Venezuela, Gobierno Bolivariano de Venezuela, disponible en: http:// 
mente a todos el territorio nacional; la segunda fase o Barrio Adentro II, comenzó en 2004 cuando se conformó un segundo nivel de atención a través de servicios diagnósticos, consultas especializadas y emergencias provenientes de Barrio Adentro I; la tercera fase o Barrio Adentro III se inició a finales del 2005 y tenía como propósito la modernización de la red hospitalaria existente en el país; la última etapa hasta el presente es Barrio Adentro IV, iniciada en septiembre del 2007 consiste en la ampliación de la red hospitalaria especializada para el fortalecimiento del sistema público nacional de salud.

Para su funcionamiento esta Misión requirió del concurso activo de las comunidades organizadas, por lo que en cada sector donde iban a funcionar los consultorios médicos se constituyeron comités de salud; instancias de apoyo definidas como organizaciones de base comunitaria "que facilita (sic) la participación de la población en la planificación, ejecución y evaluación de las actividades de salud y calidad de vida...", ${ }^{35}$ los miembros de las comunidades, en principio, eran elegidos en asambleas populares de vecinos para acompañar a los médicos en sus actividades preventivas y educativas y progresivamente, pasaron a ser consideradas como cogestionadoras de los consultorios.

Pese a la profusión de información —académica o no- cuesta dilucidar con exactitud el rol exacto que cumplen estos comités dentro del sistema público nacional, para el sector oficial, son una instancia multifuncional con responsabilidades que van desde la construcción de infraestructura física hasta llegar a todo lo que conlleva la garantía del derecho, incluida la prestación del servicio de atención a la salud; algunos estudiosos del tema y organizaciones de derechos humanos han mostrado reservas sobre las formas en que se ha propiciado la participación comunitaria por parte del Estado a fin de evitar, por una parte, la repetición de prácticas clientelares de fines del siglo pasado y por la otra, que el apoyo comunitario se convirtiera en un mecanismo para que el Estado eludiera sus obligaciones con los ciudadanos. ${ }^{36}$

En lo que se ha llamado el sistema de salud venezolano ${ }^{37}$ existe de un elevado parcelamiento de entes, recursos y sistemas de atención que funcionan

ceims.mppre.gob.ve $/$ index.php?option $=$ com_content\&view $=$ article $\mho i d=39$ :mision-barrio-adentro-i-ii-iiiivesatid $=23$ : misiones-bolivarianas $\&$ Itemid $=66$.

35 González, F., op. cit., p. 10.

36 Convite y Observatorio Comunitario por el Derecho a la Salud, op. cit.

37 Sobre este asunto es pertinente señalar que el Área de Desarrollo y Salud del Cendes$\mathrm{UCV}$, del que forma parte la autora de este trabajo, sostiene que en Venezuela no se puede 
en paralelo e independencia administrativa y funcional unos de otros; tres ejemplos dan cuenta de lo antes dicho: el Ministerio del Poder Popular para la Salud regula la red de atención primaria; el Instituto Venezolano de los Seguros Sociales, de los trabajadores asegurados, y la Misión Barrio Adentro, de una parte de la atención primaria. Durante los últimos años el enfoque preventivo se ha venido debilitado progresivamente y se ha enfatizado el médico-curativo.

La Constitución de la República Bolivariana de Venezuela asigna al Estado la obligación de garantizar el derecho a la salud, en atención a la cual debe: a) promover y políticas que eleven la calidad de vida, el bienestar colectivo y el acceso a los servicios; $b$ ) crear un sistema público nacional de salud, sobre el que se ejercerá su rectoría, con énfasis en la promoción de la salud, c) la prevención de enfermedades, su tratamiento oportuno y rehabilitación, y d) desarrollar políticas dirigidas a la formación de profesionales en la materia y a la producción de insumos para la salud, desde la industria nacional. No obstante, el examen de la realidad evidencia un deterioro de la situación de la salud en Venezuela, reconocido y denunciado por diferentes actores. ${ }^{38}$

En 2009, el entonces presidente Chávez se vio obligado a declarar la emergencia en el área; ${ }^{39}$ cuatro años más tarde, el 3 de marzo de 2013, su vicepresidente, Nicolás Maduro, afirmó que muchos hospitales del país se encontraban en una "situación bastante deplorable" 40 y, en diciembre de ese mismo

hablar de un sistema nacional de salud, ya que en realidad lo que existe es una multiplicidad de órganos y prestadores de servicios, públicos y privados que se manejan de manera inconexa y fragmentada, sin la unicidad de criterios que debe existir para hablar de un sistema, en tanto un cuerpo orgánico y estructurado.

38 Véase D’Elía, Jo, “Situación de la salud en Venezuela”, Revista SIC, núm. 769, 2014, pp. 388-391; González, Marino, Las politicas de salud en Venezuela: alternativas para su mejoramiento, Caracas, Ildis, 2008; Naranjo, María, "La política de salud en Venezuela durante el período 1999- 2012. Aportes para su formulación e implementación”, Comunidady Salud, vol.12, núm. 2, 2014, pp.80-86; Federación Médica de Venezuela, "Diagnóstico del sector salud en Venezuela: estudios de las enfermedades emergentes y reemergentes", ponencia central presentada en la LXIII Reunión Ordinaria de la Asamblea, realizada en Punto Fijo, Estado Falcón, 27 a 31 de octubre de 2008; Walter, Carlos, "A Strange Form of Declaring a Health Emergency: The Case of Venezuela”, Medical Journal Word, vol. 55, 2009, pp.157-159.

39 En Consejo de Ministros ampliado realizado en el estado Guárico señaló: "En lo social tenemos una emergencia: la salud. Declarémonos en emergencia todos", disponible en: $h t t p: / /$ www.eluniversal.com/2009/09/24/pol, consultado el 16 de abril de 2015.

40 Noticia publicada en la prensa venezolana: “informó este sábado [el vicepresidente Maduro] que el gobierno Bolivariano decidió intervenir el Hospital Periférico de Coche, en 
año, ya investido como presidente, Maduro recalcó la necesidad de: "recuperar los viejos hospitales públicos, cuyo estado calificó como «una vergüenza para la Revolución»". ${ }^{41}$

Las organizaciones de derechos humanos han reportado de manera sistemática, interrupciones en la entrega de tratamientos para enfermedades crónicas, suspensión de la radioterapia para enfermos con cáncer, déficit de especialistas para atenderlas y de medios para el diagnóstico temprano, fallas en la calidad de atención, desabastecimiento e insuficiencia de insumos y medicamentos, al punto de pasar "de desabastecimiento a escasez en productos esenciales". ${ }^{42}$ También han denunciado el deterioro de las estructuras de los servicios médicos y hospitalarios: "en los 10 principales centros hospitalarios de Caracas, 69\% de los quirófanos y 52\% de las camas no se encontraban operativas". ${ }^{43} \mathrm{La}$ insuficiencia de recursos humanos se ha estado produciendo por dos vías; por una parte, las escuelas de medicina gradúan a pocos profesionales o especialistas y, por la otra, los profesionales con alguna especialidad una vez terminada la residencia migran del sistema público al privado o hacia mejores plazas en el extranjero, lo que reduce la capacidad de dar respuesta al crecimiento de la demanda en materia de asistencia sanitaria. ${ }^{44}$

Sanitaristas, epidemiólogos y otras especialistas en salud pública han venido produciendo informes sobre el resurgimiento de enfermedades que se creían desaparecidas y nuevamente emergieron en los últimos años (Chagas,

Caracas, por irregularidades constatadas en este centro asistencial: «Hemos decidió intervenir el hospital de Coche para ir a un proceso profundo de reestructuración... será revisado lo relativo a la infraestructura, dotación de equipos y de personal, ya que se requiere médicos de diversas especialidades, sobre todo pediatras...»"(Ballesteros, Andrea, "Informó Nicolás Maduro. Gobierno Nacional anuncia intervención del Hospital Periférico de Coche", Correo del Orinoco, Venezuela, 2013, disponible en: http://mww.correodelorinoco.gob.ve/gobierno-nacionalintervino-hospital-periferico-coche/.

41 Programa Venezolano de Educación-Acción en Derechos Humanos (Provea). Informe Anual 2013: Situación de los derechos humanos en Venezuela, Venezuela, Provea, 2013. p. 197, disponible en: http:// wmw.derechos.org.ve/publicaciones/infanual/index. htm, consultado el 24 de mayo de 2016.

42 Idem.

43 Ibidem, pp. 203 y 204.

44 D'elía, Yolanda y Cabezas, Luis, Las Misiones sociales en Venezuela, Caracas, Instituto Latinoamericano de Investigaciones Sociales (Ildis), Convite A.C., 2008, disponible en: www.ildis. org.ve/, consultado el 4 de agosto de 2010. 
dengue, malaria, entre otras), ${ }^{45}$ producto —entre otras razones — de una reducción de los programas de vacunación, lo que ha facilitado: "la reaparición de brotes epidémicos de enfermedades transmisibles, controlables mediante vacunas y el riesgo de aparición de otras con grave impacto epidémico como poliomielitis y difteria que fueron erradicadas hace muchos años". ${ }^{46} \mathrm{La}$ Sociedad Venezolana de Salud Pública ${ }^{47}$ reportó que para el año 2010, en un brote que duró "más de 16 meses", el dengue alcanzó la cifra "histórica máxima de incidencia acumulada 124.931 casos con una tasa de incidencia anual acumulada de 433 x 100.000 h. de casos graves 10.278 y de muertes 124 (estimado) que afectó todo el país" y, para 2013, la malaria alcanzó: "cifras record de incidencia de casos 76.621 y tasa de 281,4 x 100.000 h., desde que se llevan registros epidemiológicos de la enfermedad con 50,1\% de aumento al comparar con el año 2012". ${ }^{48}$ El Boletín Epidemiológico emanado del Ministerio del Poder Popular para la Salud, fuente oficial del Estado venezolano en la materia, ${ }^{49}$ aporta significativas evidencias; así, en la semana 13 del primer trimestre de 2010, se notificaron 1.088 casos probables de dengue, siendo

45 Para 2010, en el país hubo un brote de mal de Chagas producto de lo cual falleció una persona, véase Cámara de Comercio de Maracaibo, Reflexiones sobre el sistema de salud venezolano, 2011, trabajo elaborado por un equipo interdisciplinario bajo el auspicio de la Cámara de Comercio de Maracaibo y presentado en diciembre de 2011, disponible en: www.ccm.org.ve/archivos/ documentos/, consultado el 14 de abril de 2016.

46 Federación Médica de Venezuela, "Diagnóstico del sector salud en Venezuela: estudios de las enfermedades emergentes y reemergentes", ponencia central presentada en la LXIII Reunión Ordinaria de la Asamblea, realizada en Punto Fijo, Estado Falcón, 27 a 31 de octubre de 2008, p.144.

47 Sociedad científica sin fines de lucro, fundada en 1952, que promueve la calidad de vida, la salud y la prevención de los factores de riesgo y daño a la salud en todo el país.

48 Sociedad venezolana de salud pública (SVSP). Defendamos la epidemiología nacional. Pequenas picaduras, grandes amenazas. Breve relato de éxitos, fracasos y amenazas de la lucha contra las enfermedades vectoriales en Venezuela, Venezuela, SVSP, 7 de abril de 2014, p. 3, disponible en: bttp:/ / wmm. bitacoramedica.com/, consultado el 30 de abril de 2016.

49 La información estadística y reportes oficiales, sobre estas y otras materias de salud pública, ha venido siendo restringida paulatinamente por parte de las autoridades competentes. Desde el año 2012 fueron suspendidos los anuarios de Mortalidad y Morbilidad, y el Boletín Epidemiológico estuvo en esa misma situación desde noviembre de 2014 hasta febrero de 2016. El mencionado instrumento tiene carácter obligatorio para el Estado, quien debe emitirlo, periódicamente, con la lista de las enfermedades que circulen en el país y sus posibles amenazas, para los efectos de orientar tanto a la ciudadanía como a los profesionales de la salud, de manera técnica y confiable, en el reconocimiento de los factores de riesgo de las enfermedades; así como, para ayudar a la toma de decisiones sobre políticas sanitarias, véa- 
que la tasa nacional de incidencia acumulada para esa semana fue de 76.8 por 100.000 habitantes, la cual era superada por 9 estados. Para la semana 14 del año 2014, el acumulado fue 75.020 casos y el índice epidémico fue de 164, lo que representa un incremento de 64\% "con relación a la mediatriz 20092013". Hasta esa fecha existía "un acumulativo de 73.572 casos con origen de infección en el país", lo que representaba un "aumento de $12 \%$ con respecto al período homólogo del año anterior"; en el caso de la malaria, para 2013, se reportó un acumulativo de 12.717 casos. ${ }^{50}$

\section{A MANERA DE CIERRE}

A partir de las propuestas de diversos sectores de la sociedad venezolana, la Constitución promulgada en 1999 postula una ciudadanía más inclusiva con mecanismos de participación ciudadana y una ampliación de los derechos sociales; ratificando así, la concepción presente en los instrumentos internacionales sobre los derechos humanos: se promueve la igualdad de todos los integrantes de la sociedad venezolana y la corresponsabilidad: los ciudadanos deben participar activamente en el control de la gestión de su sistema de derechos. Bajo ese marco filosófico, el derecho a la salud tiene un carácter marcadamente universalista: la salud se tutela como parte del derecho a la vida; su promoción, defensa y gestión se convierte en un asunto de todos, se insta a la construcción de políticas que respeten al ciudadano, en tanto sujeto de derechos y permitan cambios en su calidad de vida, bienestar y procuren su acceso a los servicios.

En los hechos, las estructuras y mecanismos institucionales desarrollados para hacer efectivo el derecho a la salud han suscitado dudas, tanto en medios académicos, como en los ciudadanos organizados en la defensa de los derechos humanos por la repetición de esquemas pasados y, el creciente deterioro de las condiciones de acceso a servicios, tratamientos y medicamentos lo que,

se: http:// www.eluniversal.com/noticias/estilo-vida/ reaparecen-incompletos-los-boletines-epidemiologicos, consultado el 16 de abril de 2016.

50 Ministerio del Poder Popular para la Salud, Boletín Epidemiológico, Venezuela, Ministerio de Poder Popular para la Salud, 2014, disponible en: http://mmm.mppp.gob.ve/wp-content, consultado el 12 de junio de 2017, disponible en: http://wmw.bvs.gob.ve/ y http://mwm.bus.gob.ve/ boletin_epidemiologico/2010, consultado el 30 de abril de 2016. 
entre otros factores dificulta las oportunidades reales de los ciudadanos de hacer efectivo su derecho.

La realización del derecho a la salud suele depender de la naturaleza, calidad, intensidad, oportunidad y pertinencia de las políticas públicas en la materia; para darle contenido y efectividad al derecho, para hacerlo real, se requieren cambios sustantivos que abarquen la organización del sistema de salud, de los programas y servicios que se presten, así como un adecuado financiamiento con verdaderos criterios de universalidad, equidad y eficacia. Conforme a lo señalado en las páginas iniciales de este artículo las reglas, mecanismos, instancias, contextos, recursos y actores resultan imprescindibles para definir y realizar, de manera efectiva, el derecho. En el caso venezolano, no ha habido la sinergia necesaria entre esos factores y se ha producido un abismo entre la concepción del derecho y su realización, con consecuencias negativas en la integración social, el consumo, la salud, la concepción del derecho y por ende, en el ejercicio de la ciudadanía.

\section{REFERENCIAS}

Asamblea nacional Constituyente, Debates de la Constitución de 1999 (versión en corrección), Caracas, Servicio Autónomo de Información Legislativa, 1999.

ALAYÓN M., Rubén, "Barrio Adentro: combatir la exclusión profundizando la democracia", Revista Venezolana de Economía y Ciencias Sociales, vol.11, núm. 3, septiembre-diciembre de 2005.

Alvarado, Neritza, "Populismo, democracia y política social en Venezuela", Fermentum, Mérida, año 15, núm. 44, septiembre-diciembre de 2005.

Alvarado, Neritza, "Pobreza y exclusión en Venezuela a la luz de las Misiones Sociales (2003-2004)", Fermentum, Mérida, año 14, núm. 39, enero-abril de 2004.

Alvarado, Neritza, "La atención a la pobreza en Venezuela del 'Gran Viraje’ a la 'V República', 1989-2002”, Revista Venezolana de Análisis de Coyuntura, vol. IX, núm. 2, julio-diciembre de 2003.

Alvarado, Neritza, "Pobreza y asistencialismo en Venezuela", Revista de Ciencias Sociales, vol. IX, diciembre de 2003. 
Ballesteros, Andrea, "Informó Nicolás Maduro. Gobierno Nacional anuncia intervención del Hospital Periférico de Coche", Correo del Orinoco, Venezuela, 2013, disponible en: http://www.correodelorinoco.gob.ve/gobiernonacional-intervino-hospital-periferico-coche/.

BREWER-CARíAs, Allan, La Constitución de 1999, Caracas, Editorial Arte, 2000.

Castellanos, Pedro Luis, "Notas sobre el Estado y la salud en Venezuela", Cuadernos de la sociedad venezolana de planificación, Caracas, núm. 156-158, 1982.

COLMENARES, Isabel R. et al., "La política de salud en Venezuela en el gobierno de Hugo Chávez”, Saber, Venezuela, vol. 17, núm. 2, 2005, disponible en: wnw.ildis.org.ve/, consultado el 10 de noviembre de 2014.

Contreras, Juana, El Plan Barrio Adentro. Ponencia presentada en el seminario nacional: política social, ¿un nuevo paradigma?, Caracas, FEGS, 2004.

CONVITE y ObSERVATORIO COMUNITARIO POR EL DERECHO A LA SALUd, Informe sobre el derecho a la salud en Venezuela 2007. Situación del derecho a la atención sanitaria, 2007, disponible en: wmw.venescopio.org.ve, consultado el 27 de abril de 2008.

COMISIÓN DE ENLACE PARA LA INTERNACIONALIZACIÓN DE LAS MiSIONES Sociales, Misión Barrio Adentro I, II, III, IV, Venezuela, gobierno Bolivariano de Venezuela, disponible en: http://ceims.mppre.gob.ve/index. php? option $=$ com_contenteview $=$ article $\delta i d=39$ :mision-barrio-adentro-i-ii-iiiiveratid=23:misiones-bolivarianas 2 Itemid $=66$.

COMisión PREsidencial PARA la REFORMA DEL Estado, Una politica social para la reafirmación de la democracia, Caracas, Editorial Arte, 1989, vol. 8.

"Decreto de la Presidencia de la República, núm. 2745, 2004", Gaceta Oficial, núm. 37.865, 26 de enero de 2004.

"Decreto de la Presidencia de la República, núm. 3, 1999”, Gaceta Oficial, núm. 36.334, 2 de febrero de 1999.

D’ELÍA, Jo, "Situación de la salud en Venezuela", Revista SIC, 769, 2014, disponible en: http: / / wmw.ildis.org.ve/ website/ administrador, consultado el 15 de abril de 2016.

D’ELÍA, Yolanda, “Cambiando la orientación de las políticas públicas hacia el impacto en la calidad de vida desde una perspectiva de derechos y equidad", Informe social, Caracas, Ildis-Fundación Friedrich Ebert, 2002. 
D’ELÍA, Yolanda y CABEZAS, Luis, Las Misiones sociales en Venezuela, Caracas, Instituto Latinoamericano de Investigaciones Sociales (Ildis), Convite A.C. 2008, disponible en: www.ildis.org.ve/, consultado el 4 de agosto de 2010.

Díaz Polanco, Jorge, Saludy hegemonía en Venezuela: Barrio Adentro, continente afuera, Caracas, Cendes-UCV, 2008.

FARÍAS-SUÁREZ, Adriana y FARÍAS de EsTANY, Jenny, "Participación comunitaria: otra mirada al sistema de salud venezolano", Población y Salud en Mesoamérica, San José, vol. 7, núm. 1, julio-diciembre de 2009, disponible en: wmw.ccp.ucr.ac.cr., consultado el 20 de octubre de 2009.

Federación Médica de Venezuela, "Diagnóstico del sector salud en Venezuela: estudios de las enfermedades emergentes y reemergentes”, ponencia central presentada en la LXIII Reunión Ordinaria de la Asamblea, realizada en Punto Fijo, Estado Falcón, 27 al 31 de octubre de 2008, disponible en: http:/ / mww.ovsalud.org/doc, consultado el 14 de abril de 2016.

FEO, Oscar, "La salud en la nueva Constitución", en MAINGON, Thais (coord.), La cuestión social en la Constitución bolivariana de Venezuela, Caracas, Cendes-UCV, 2000, serie Temas de Docencia.

FEO, Oscar y CurCiO, Pascualina, "La salud en el proceso constituyente venezolano", Revista Cubana Salud Pública, La Habana, vol. 30, núm. 2, jun, 2004, disponible en: wmm.scielo.sld.cu, consultado el 29 mayo de 2012.

GÓMEZ SÁNCHEZ, Irey y ALARCÓN FlORES, Luis, "Política social y construcción de ciudadanía en Venezuela", Multiciencias, vol. 9, núm. 2, 2009, disponible en: wmm. revistas.luz.edu.ve/, consultado el 25 de noviembre de 2011.

GONZÁLEZ, Lissette, La política social en Venezuela, Caracas, Fundación Centro Gumilla, 1996, Curso de Formación sociopolítica 35.

GONZÁLEZ, Lissette y LACRUZ, Tito, La política social en Venezuela, Caracas, Centro Gumilla, 2008.

GONZÁLEZ, Marino, Las politicas de salud en Venezuela: alternativas para su mejoramiento, Caracas, Ildis, 2008;

GONZÁLEZ FERNÁNDEZ, José Daniel, Misión Barrio Adentro: programa de atención, Inclusión y Equidad en Salud, Maracay, IAESP "Dr. Arnoldo Gabaldón” (Mimeo), 2005. 
Kornblith, Miriam y Maingon, Thais, Estado y Gasto Público en Venezuela, 1936-1980, Caracas, Ediciones de la Biblioteca de la Universidad Central de Venezuela, 1985.

Maingon, Thaís, "Política social en Venezuela: 1999-2003", Cuadernos del Cendes, Caracas, 2003, núm. 55.

MAINGON, Thaís, Un siglo de politica de salud en Venezuela: eficiencia, eficacia y equidad, tesis doctoral, Facultad de Ciencias Políticas y Jurídicas, Universidad Central de Venezuela.

MÁrquez, Trino, El Estado social en Venezuela, Caracas, Ediciones del Congreso de la República, 1992.

MÉNDez CEgARRA, Absalón, Estado, politica social y trabajo social en la Venezuela actual, Caracas, Universidad Central de Venezuela, Facultad de Ciencias Económicas y Sociales, Unidad de Publicaciones, 1992.

Mujica Chirinos, Norbis, "Estado y políticas sociales en Venezuela, cla Quinta República o el regreso al pasado?", Revista Venežlana de Gerencia, año 7, núm. 18, 2002.

Mujica Chirinos, Norbis y Alvarado, Neritza, "Pobreza y política social en venezuela hoy: reflexiones sobre su concepción y praxis", Revista BCV, Foros 1, Caracas, abril de 2004.

NARANJo, María, "La política de salud en Venezuela durante el período 19992012. Aportes para su formulación e implementación”, Comunidady salud, vol. 12, núm. 2, 2014.

Organización Panamericana de la SAlud, Barrio Adentro: derecho a la salud e inclusión social en Venezuela, Caracas, 2006.

Programa Venezolano de Educación-Acción en Derechos HumaNOS (PROVEA), Informe anual 2013: situación de los derechos bumanos en Venequela, 2013, disponible en: ww.derechos.org.ve/publicaciones/infanual/, consultado el 24 de mayo de 2016.

República Bolivariana de Venezuela, Boletin Epidemiológico, Ministerio del Poder Popular para la Salud, 2014, disponible en: http:/ / mmw.bvs.gob.ve/ y http://wnw.bvs.gob.ve/boletin_epidemiologico/2010, consultado el 30 de abril de 2016.

República Bolivariana de Venezuela, Lineas generales del Plan de Desarrollo Económico y Social de la Nación 2001-2007, Ministerio del Poder Popular 
para la Planificación y el Desarrollo, 2001, disponible en: http:/ / www.mppp. gob.ve/wp-content, consultado el 12 de junio de 2017.

República Bolivariana de Venezuela, "Asamblea Nacional Constituyente. Constitución de la República Bolivariana de Venezuela”, Gaceta Oficial, Caracas, núm. 5.453, extraordinario, 24 de marzo de 1999.

Sociedad Venezolana de SAlud Pública, Defendamos la Epidemiología Nacional. Pequeñas picaduras, grandes amenazas. Breve relato de éxitos, fracasos y amenazas de la lucha contra las enfermedades vectoriales en Venezuela, 7 de abril de 2014, disponible en: http:// wmw.bitacoramedica.com/, consultado el 30 de abril de 2016.

Uharte POZAs, Luis Miguel, Politica social en Venezuela: ¿un nuevo paradigma? tesis doctoral, Universidad Complutense de Madrid, 2008.

Villasana, Pedro, 'De Alma Ata a Barrio Adentro. Una aproximación al sentido histórico de las metamorfosis del discurso de la participación en salud en Venezuela", en LÉvY, Johanna y MALO, Miguel (eds.), De la participación en salud a la construcción del poder popular: experiencias para el debate, Maracay, IAES “Dr. Arnoldo Gabaldón, 2010.

WALTER, Carlos, "A Strange Form of Declaring a Health Emergency: The Case of Venezuela", Medical Journal Word, vol. 55, 2009, disponible en: http:// wnm.wma.net/en/30publications, consultado el 16 de abril de 2016. 